\title{
Experimental Quantum Error Detection
}

\author{
Xian-Min Jin ${ }^{1,2,3}$, Zhen-Huan $\mathrm{Yi}^{4}$, Bin Yang ${ }^{1}$, Fei Zhou ${ }^{4}$, Tao Yang ${ }^{1}$ \& Cheng-Zhi Peng ${ }^{1,4}$
}

SUBJECT AREAS:

QUANTUM PHYSICS

APPLIED PHYSICS

QUANTUM OPTICS

FIBRE OPTICS AND OPTICAL COMMUNICATIONS

Received

25 May 2012

Accepted

16 August 2012

Published

4 September 2012

Correspondence and requests for materials should be addressed to

X.M.J. (x.jin1@

physics.ox.ac.uk)
${ }^{1}$ Hefei National Laboratory for Physical Sciences at Microscale and Department of Modern Physics, University of Science and Technology of China, Hefei, Anhui 230026, PR China, ${ }^{2}$ Clarendon Laboratory, Department of Physics, University of Oxford, Oxford OXI 3PU, United Kingdom, ${ }^{3}$ Department of Physics, Shanghai Jiao Tong University, Shanghai 200240, PR China, ${ }^{4}$ Physics Department, Tsinghua University, Beijing 100084, PR China.

Faithful transmission of quantum information is a crucial ingredient in quantum communication networks. To overcome the unavoidable decoherence in a noisy channel, to date, many efforts have been made to transmit one state by consuming large numbers of time-synchronized ancilla states. However, such huge demands of quantum resources are hard to meet with current technology and this restricts practical applications. Here we experimentally demonstrate quantum error detection, an economical approach to reliably protecting a qubit against bit-flip errors. Arbitrary unknown polarization states of single photons and entangled photons are converted into time bins deterministically via a modified Franson interferometer. Noise arising in both $10 \mathrm{~m}$ and $0.8 \mathrm{~km}$ fiber, which induces associated errors on the reference frame of time bins, is filtered when photons are detected. The demonstrated resource efficiency and state independence make this protocol a promising candidate for implementing a real-world quantum communication network.

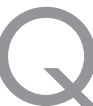

uantum error correction is an extremely elegant solution to the problem of noise in a quantum channel. It allows to protect a fragile quantum state by encoding the state into a multi-particle entangled state ${ }^{1-4}$. Errors affecting the quantum system can be then detected and corrected. So far, significant experimental progress has been achieved in the nuclear magnetic resonance ${ }^{5-7}$, ion-trap ${ }^{8}$ and linear optics. Another original approach is quantum purification, ${ }^{10-12}$ which can extract a certain number of almost perfectly pure states out of a large number of less-pure states using local operation and classical communication. Furthermore, some great efforts have been made to protect the transmission of a quantum state against a specific error using fewer ancillary particles, such as schemes employing decoherence-free subspaces for collective noise ${ }^{13-17}$, quantum error rejection for bit-flip error (BFE) $)^{18,19}$ and quantum error filtration for phase-shift error (PSE) ${ }^{20,21}$.

The main obstacle for a large-scale implementation of these schemes is that the success probability decays as $\eta_{\text {eff }}=\lambda^{1+N} \cdot\left(1-\eta_{\text {loss }}\right)^{1+N}$, i.e. exponentially with both the generation probability $\lambda$, and the decoherence-induce loss $\eta_{\text {loss }}$ for a number $N$ of ancillas. $\lambda$ and $1-\eta_{\text {loss }}$ in principle can be improved to 1 , by generating photons deterministically or synchronizing probabilistic photonic sources with quantum memories, and preventing photons from coupling with environment. This is far beyond what is experimentally possible at present. It therefore remains challenging to implement the above schemes in a real-world application, such as quantum error-free transmission over long distance ${ }^{22}$.

To overcome the aforementioned problems, Kalamidas et al. proposed a quantum error detection scheme ${ }^{23}$ in which path information of photons was used to detect BFE on polarization qubit, without using ancilla photons. As a step forward in this direction, in this letter, we propose and experimentally demonstrate a modified version of quantum error detection by employing time information of photons, which is stable and benefits from the simplicity of using time-bin configuration ${ }^{24,25}$. Unlike the quantum error filtration scheme $e^{20,21}$, which was designed to protect phase qubits, the aim of the quantum error detection scheme is to protect polarization qubits without using an ancilla, thereby confirming the feasibility of faithfully transferring polarization qubits in fiber channel. Experimentally, comparing with the original quantum error detection scheme ${ }^{23}$, our modified scheme in which only one fiber channel is involved is less sensitive to environment and cost-efficient, therefore more suitable for real-world applications.

\section{Results}

Without loss of generality, let us consider the scenario in which Alice wants to send an arbitrary polarization state $|\psi\rangle=\alpha|\mathrm{H}\rangle+\beta|\mathrm{V}\rangle$ to Bob via a noisy quantum channel, where $\alpha$ and $\beta$ are two complex numbers satisfying $|\alpha|^{2}+|\beta|^{2}=1,|H\rangle(|V\rangle)$ denotes horizontal (vertical) linear polarization. As shown in Fig. 1a, instead of directly sending the initial state to Bob, Alice encodes it into two time bins with specified polarization using an unbalanced 
a

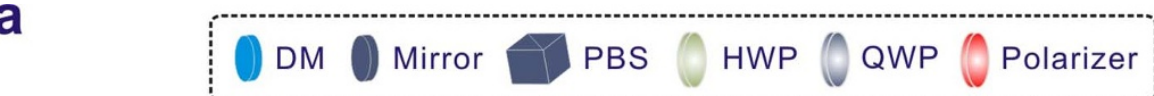

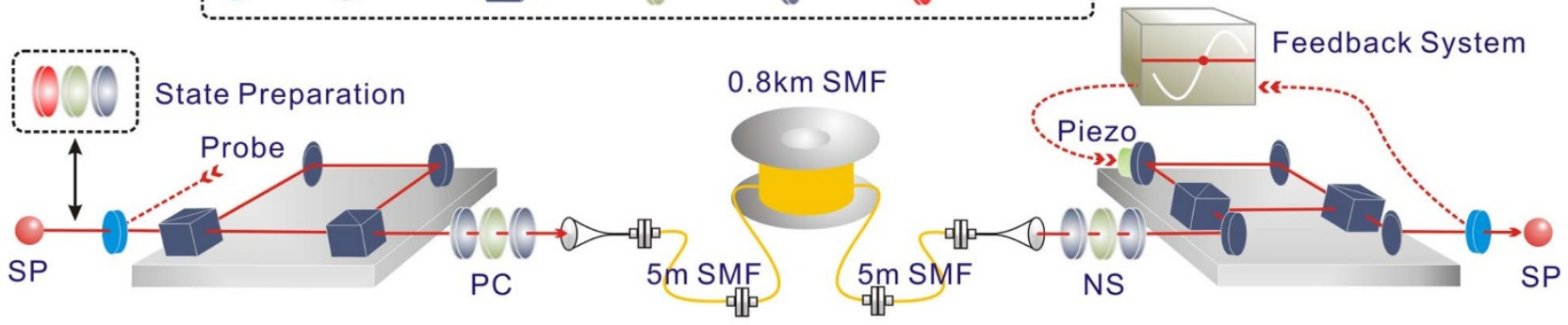

b
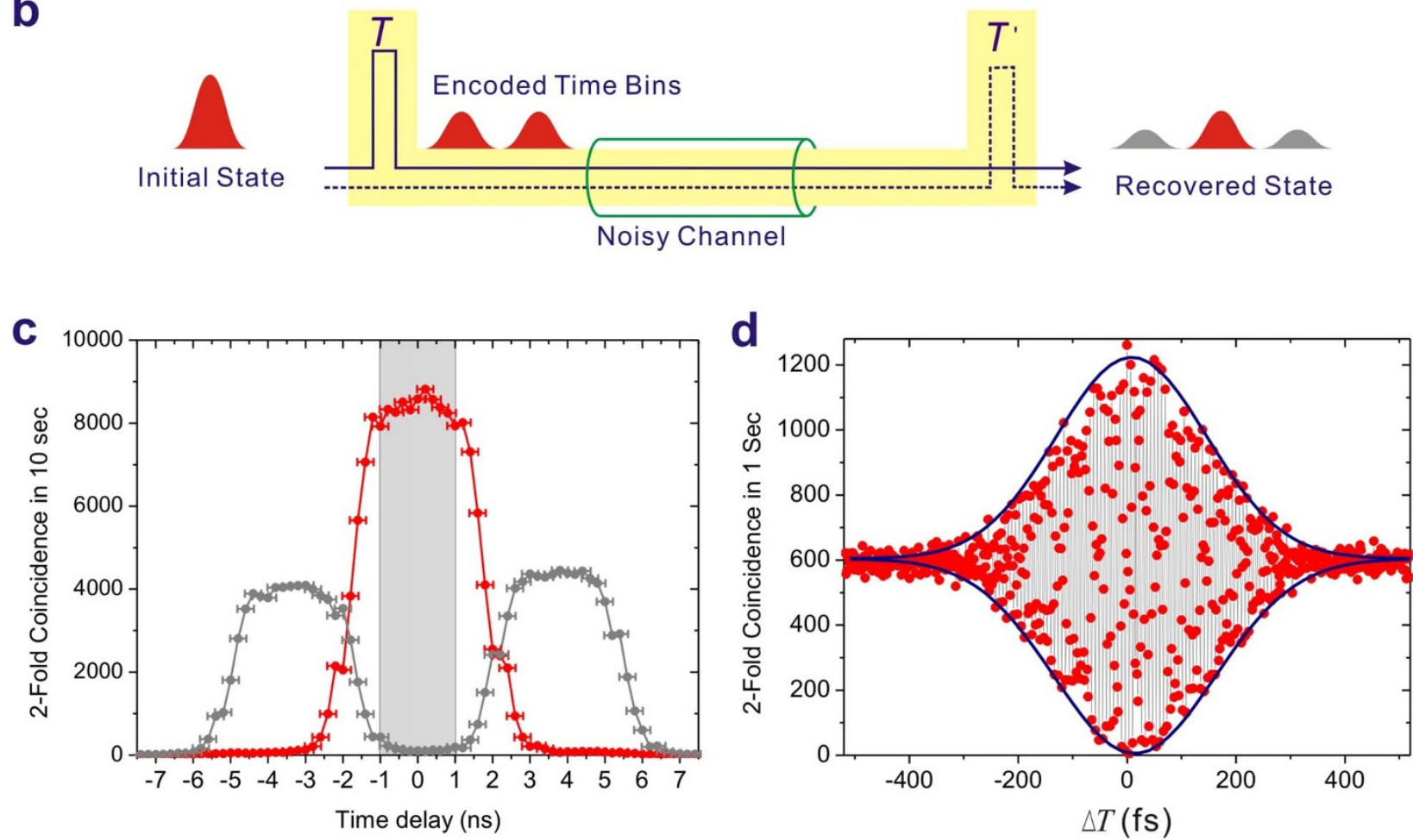

Figure $1 \mid$ Schematic of quantum error detection. (a) Experimental setup. The state-carrier photon (SP) is guided into the unbalanced Mach-Zehnder interferometer $\left(\mathrm{UMZI}_{1}\right)$ and its $V$ mode is delayed about $2.965 \mathrm{~ns}$. The noisy channel consists of a polarization controller (PC), $10 \mathrm{~m}$ or $0.8 \mathrm{~km} \mathrm{SMF}$, and a noise simulator (NS). $\mathrm{UMZI}_{2}$ introduces a time delay of $2.965 \mathrm{~ns}$ on the $H$ mode of SP. The probe laser $(633 \mathrm{~nm})$ is coupled into and out of the system by two dichroic mirrors (DM). PBS: polarizing beamsplitter, HWP: half-wave plate, QWP: quarter-wave plate, Piezo: piezoelectric actuator. (b) principle diagram of quantum error detection showing evolution of the quantum state. The solid line denotes the $V$ mode of SP and the dashed denotes the $H$ mode. (c) Observed three peaks corresponding to the interval of $\Delta t, \Delta t+T$ and $\Delta t+2 T$. Error bars are given by the total time jitter of the electrical devices of photon detection and coincidence system. (d) Observed interference pattern by measuring the 2 -fold coincidence between TP and SP. $\Delta T$ is the time delay introduced by the Piezo.

Mach-Zehnder interferometer ${ }^{26}$ (UMZI). The configuration of two polarizing beam splitters (PBS) enables $H$ mode to go on the short path and $V$ mode to go on the long path in a deterministic manner:

$$
\hat{T}|\psi\rangle=\alpha|H\rangle+\beta\left|V_{T}\right\rangle
$$

where subscript $T$ denotes the additional time delay on the $V$ mode induced by $\mathrm{UMZI}_{1}$. General error in a noisy channel can be expressed as $E=e_{0} I+e_{1} X+e_{2} Z+e_{3} X Z\left(\left|e_{0}\right|^{2}+\left|e_{1}\right|^{2}+\left|e_{2}\right|^{2}+\left|e_{3}\right|^{2}=1\right)$, where the three Pauli matrices $I=\left(\begin{array}{ll}1 & 0 \\ 0 & 1\end{array}\right), X=\left(\begin{array}{ll}0 & 1 \\ 1 & 0\end{array}\right)$ and $Z=$ $\left(\begin{array}{cc}1 & 0 \\ 0 & -1\end{array}\right)$ denote identity, BFE and PSE respectively ${ }^{27}$. At Bob's station, he applies a time delay $T^{\prime}$ on the $H$ mode with a second interferometer $\mathrm{UMZI}_{2}$. The output state with specific error can be written as:

$$
\begin{gathered}
\hat{T}^{\prime} I \hat{T}|\psi\rangle=\alpha\left|H_{T^{\prime}}\right\rangle+e^{i \phi_{1}} \beta\left|V_{T}\right\rangle \\
\hat{T}^{\prime} X \hat{T}|\psi\rangle=\alpha|V\rangle+e^{i \phi_{2}} \beta\left|H_{T T^{\prime}}\right\rangle \\
\hat{T}^{\prime} Z \hat{T}|\psi\rangle=\alpha\left|H_{T^{\prime}}\right\rangle-e^{i \phi_{1}} \beta\left|V_{T}\right\rangle \\
\hat{T}^{\prime} X Z \hat{T}|\psi\rangle=\alpha|V\rangle-e^{i \phi_{2}} \beta\left|H_{T T^{\prime}}\right\rangle
\end{gathered}
$$

where $\phi_{1}=k c\left(T-T^{\prime}\right), \phi_{2}=k c\left(T+T^{\prime}\right), k$ is the wave vector and $c$ is the speed of light.

The two time bins in equation (2b) or (2d) are so widely separated (larger than the coherence time of the state-carrier photon (SP)) that the polarization state decays into the mixed state $|\alpha|^{2}|V\rangle\langle V|+$ $|\beta|^{2}\left|H_{T T^{\prime}}\right\rangle\left\langle H_{T T^{\prime}}\right|$, giving rise to the two side peaks in Fig. 1b. The time bins in equation $(2 \mathrm{a})$ or $(2 \mathrm{c})$ contribute to the central peak. The BFE 

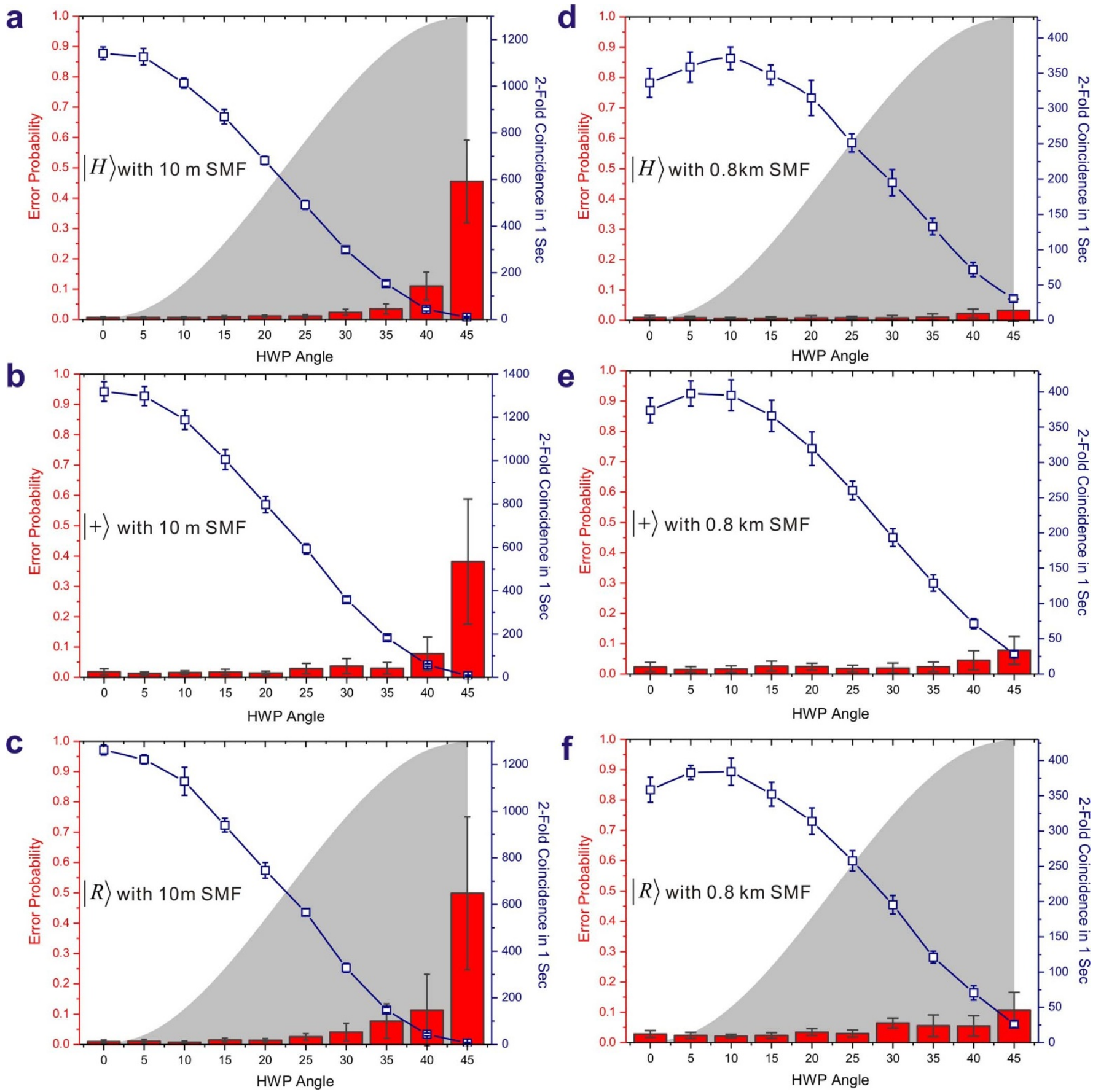

Figure $2 \mid$ Quantum error detection against BFE. The results are taken for three complementary bases, linear polarization $|H\rangle$, $|+\rangle$ and circular polarization $|R\rangle$. (a), (b), (c) The performance in the case of $10 \mathrm{~m} \mathrm{SMF}$. (d), (e), (f) The performance in the case of $0.8 \mathrm{~km}$ SMF. The 2-fold coincidence curves in (d), (e), (f) have a small shift about 7 degree along the axis of HWP angle, which is due to a birefringence-induced polarization rotation in the long fiber that occurred after initializing the error of the whole channel. The gray parts are the error probabilities simulated by the NS. The red histograms show the error probabilities obtained with quantum error detection. The blue squares show the efficiencies of transmission. Error bars are given by Poissonian statistics.

and bit-phase-flip errors can therefore be effectively detected when SP ends up in either of the two side peaks, meanwhile BFE-free events can be retrieved by post-selecting the central peak. This step can be realized by comparing the time interval of sending and receiving the state with the assistance of synchronization clocks. Bob can ensure the received state has no BFE if the interval is equal to $\Delta t+T$ (central peak), where $\Delta t$ is the time that the SP takes to fly from Alice to Bob following the short paths of $\mathrm{UMZI}_{1}$ and $\mathrm{UMZI}_{2}$. If the interval is $\Delta t$ or $\Delta t+2 T$ (side peaks), it means that a BFE has occurred during the transmission, Bob just needs to discard the state. This process can completely detect BFEs while reducing the transmission efficiency to $\left|e_{0}\right|^{2}+\left|e_{2}\right|^{2}$.

In our experiment, type-II spontaneous parametric down conversion $^{28}$ is employed to prepare a heralded single photon SP and polarization entanglement. A semiconductor laser beam (power $34.5 \mathrm{~mW}$, beam waist $100 \mu \mathrm{m}$ and central wavelength $405 \mathrm{~nm}$ ) incident on a $2 \mathrm{~mm}$ beta-barium-borate (BBO) crystal, generates polarization entangled pairs $(|H\rangle|V\rangle-|V\rangle|H\rangle) / \sqrt{2}$ at $810 \mathrm{~nm}^{29}$. The down-converted extraordinary and ordinary photons have different velocities and travel along different paths due to birefringence 

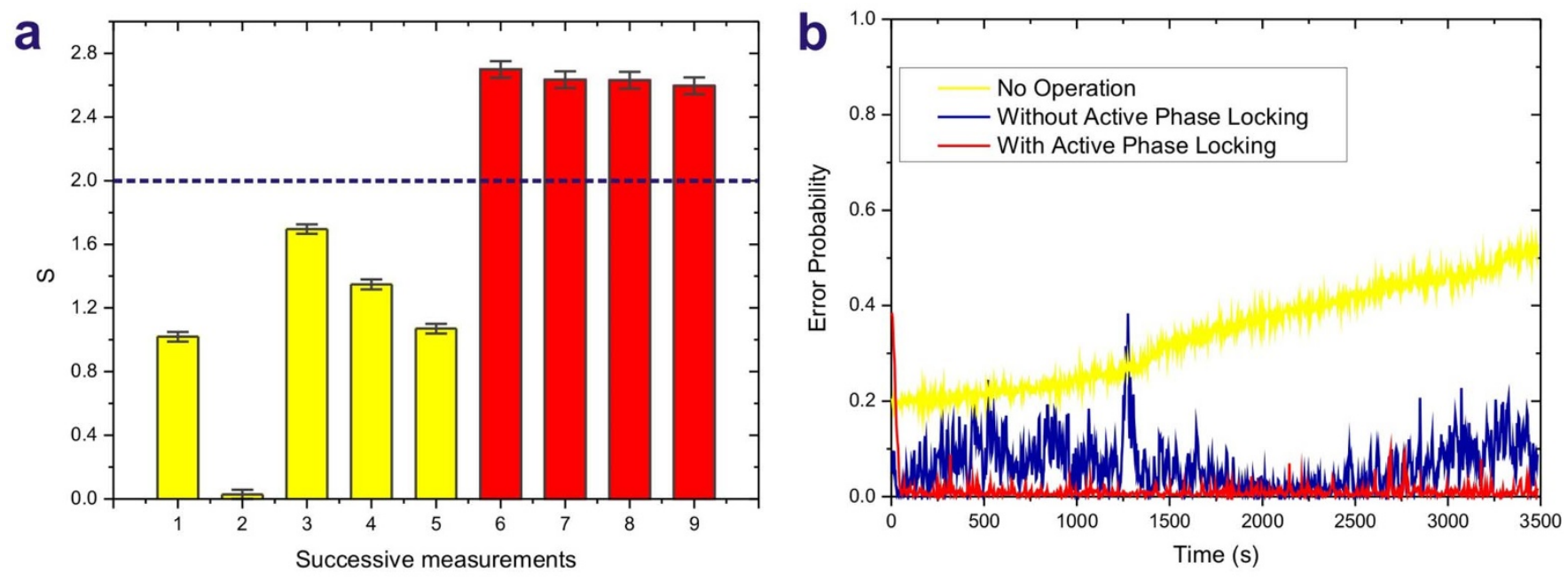

Figure 3 Entanglement distribution and system stability. (a) The experimental measurements of CHSH inequality after passing through a twisted fiber. The yellow histograms denote the measured $S$ values without error detection and the red histograms are the $S$ values taken with error detection operation. The twist is changed after each measurement. The dashed line marks the violation boundary. Error bars are given by Poissonian statistics. (b) The stability of the system with and without active phase locking. The yellow curve is obtained with neither quantum error detection nor active phase locking operation. Experimental data is collected continuously over one hour with $0.8 \mathrm{~km} \mathrm{SMF}$.

of the BBO crystal. The resulting walk-off effects are compensated by a combination of a HWP and an additional $1 \mathrm{~mm}$ BBO crystal in each arm. With single mode fibers (SMFs) and 4-nm bandwidth interference filters, we collect about 80,000 single photons per second in each arm of the source and 22,000 pairs of polarization entangled photons per second. The observed visibilities of the polarization correlations, which can reveal the quality of our prepared entanglement, are about $98.1 \%$ for the $|H\rangle /|V\rangle$ basis, and $92.6 \%$ for the $|+\rangle /|-\rangle$ basis where $| \pm\rangle=(|H\rangle \pm|V\rangle) / \sqrt{2}$. Because photons are always created in pairs, the detection of one photon indicates, or "heralds", the existence of its twin. Thus the SP with $H$ polarization can be prepared conditioning on the detection of the trigger photon (TP) with $V$ polarization.

As is shown in Fig. 1b, an arbitrary polarization state $|\psi\rangle=$ $\alpha|\mathrm{H}\rangle+\beta|\mathrm{V}\rangle$ is prepared by a set of optical components consisting of a polarizer, a half wave plate (HWP) and a quarter wave plate (QWP). The time delay $T$ introduced by $\mathrm{UMZI}_{1}$ is about $2.965 \mathrm{~ns}$. The photon subsequently passes a polarization controller (PC), two pieces of $5 \mathrm{~m} \mathrm{SMF}$, and a BFE simulator. The particular configuration of the PC, a HWP sandwiched by two QWPs, has been proved capable of unitarily realizing arbitrary polarization manipulation. Here the PC is employed to compensate and initialize the error of the whole channel. In order to see the performance of quantum error detection, a noise simulator (NS) is necessary to provide a calibrated source of BFE noise in the channel. Its structure is identical to the PC, except that the QWPs are set at a fixed angle of $90^{\circ}$. By randomly setting the HWP axis to be oriented at $\pm \theta$, a noisy quantum channel ${ }^{19}$ can be engineered with a BFE probability of $\sin ^{2}(2 \theta)$. UMZI subsequently applies a $2.965 \mathrm{~ns}$ time delay $T^{\prime}$ on the $H$ mode of the state by guiding the photon into the interferometer from the other input port, see Fig. 1a. To observe the temporal profile of the output states, we prepare the SP in the state $|+\rangle$ and measure 2 -fold coincidence rates by scanning an electronic delay between the SP and TP signals with a range of $16 \mathrm{~ns}$. We choose to observe two representative cases of $0 \%$ and $100 \%$ BFE probabilities by setting the HWP in the NS to $0^{\circ}$ and $45^{\circ}$ respectively. As expected, we obtain three peaks corresponding to the interval of $\Delta t, \Delta t+T$ and $\Delta t+2 T$. As shown in Fig. 1c, our experimental data illustrates that BFEs can be effectively detected by comparing the time interval between the detection of the $\mathrm{TP}$ and SP. The window of coincidence logic is set at $2 \mathrm{~ns}$, which is sufficient to filter out the two side peaks, so that only the events without a BFE are preserved. To ensure the two time bins at the central peak overlap coherently, the first-order interference is utilized to find and fix $\Delta T=T-T^{\prime} \approx 0$ (see Methods).

To verify the success of error detection, we select linear polarization $|H\rangle,|+\rangle$ and the circular polarization $|R\rangle=(|H\rangle+i|V\rangle) / \sqrt{2}$ as initial states to be transmitted. BFEs are quantitatively introduced from 0 to 1 by changing $\theta$ with an increment of 5 degree in the NS. We investigate the change of error probabilities and coincidence rates under these conditions with a $10 \mathrm{~m}$ fiber (see Fig. 2a, b and c). A further test for longer SMF can be realized by simply inserting $0.8 \mathrm{~km}$ fiber (see Fig. 2d, e and f). The errors in the noisy channel increase with the angle $\theta$ but the final errors on output states after quantum error detection are all confined within 10\%, except the case of $100 \%$ BFE probability. In fact, the error probabilities mainly come from the imperfection of state preparation and the limited signal-tonoise ratio. In the case of inserting $0.8 \mathrm{~km}$ fiber, the performance of error detection is very similar to the case of short fiber, representing significant robustness of our protocol, while 2 -fold coincidence curves have a small shift about 7 degree along the axis of HWP angle, which is due to a birefringence-induced polarization rotation in the long fiber that occurred after initializing the error of the whole channel. The coincidence rate depends strongly on the channel noise. It decreases to a minimum when the error probability increases close to $100 \%$. By actively introducing random BFEs on the channel, the coincidences and error probabilities can be made to depend much less on the channel errors ${ }^{14,16}$.

To verify further the state-independent feature of our error detection scheme, and thus show the capability to distribute entanglement, we feed one of the entangled photons into a polarization analyzer on Alice's side, and we send the other photon into the channel with $0.8 \mathrm{~km}$ SMF and a polarization analyzer on Bob's side. The entanglement is verified by the way of measuring the violation of the Clauser-Horne-Shimony-Holt (CHSH) inequality ${ }^{30}$. The polarization correlation function is defined as follows,

$$
E\left(\theta_{A}, \theta_{B}\right)=\frac{N_{++}+N_{--}-N_{+-}-N_{-+}}{N_{++}+N_{--}+N_{+-}+N_{-+}}
$$

Where $\mathrm{N}_{++}, \mathrm{N}_{--}, \mathrm{N}_{+-}$and $\mathrm{N}_{-+}$are the numbers of coincidence counts between Alice and Bob with the actual settings of $\left(\theta_{A}, \theta_{B}\right)$, $\left(\theta_{A}+\pi / 2, \theta_{B}+\pi / 2\right),\left(\theta_{A}, \theta_{B}+\pi / 2\right)$ and $\left(\theta_{A}+\pi / 2, \theta_{B}\right)$ respectively. In the CHSH inequality, the parameter $S$ is defined as, 


$$
S=\left|E\left(\theta_{A}, \theta_{B}\right)-E\left(\theta_{A}, \theta_{B}^{\prime}\right)+E\left(\theta_{A}^{\prime}, \theta_{B}\right)+E\left(\theta_{A}^{\prime}, \theta_{B}^{\prime}\right)\right|
$$

Classically, no matter what angles $\theta_{A}$ and $\theta_{B}$ are set to, the parameter $S$ is bounded by the constraint $S \leq 2$. Quantum mechanically, $S$ can exceed this bound, with a maximal value given by $S=$ $2 \sqrt{2} \approx 2.828$ when the polarization angles are set to $\left(\theta_{A}, \theta_{A}^{\prime}\right.$, $\left.\theta_{B}, \theta_{A}^{\prime}\right)=\left(0^{\circ}, 45^{\circ}, 22.5^{\circ}, 67.5^{\circ}\right)$. The measured value of $S$ is $S=2.70$ \pm 0.05 , which violates $\mathrm{CHSH}$ Inequality by 14 standard deviations. This clearly confirms that quantum entanglement is successfully recovered from a noisy signal using our error detection protocol. We perform the CHSH inequality measurement several times with and without quantum error detection. The Fig. 3 a shows that the CHSH inequality is violated when error detection is used, but that without error detection, the violation is lost.

In our quantum error detection system, PSE behaves as the time drift between time bins. A weak coherent laser $(633 \mathrm{~nm})$ with welldefined polarization $|+\rangle$ is employed to probe the drift by coupling in and out of the system and measuring in the $|+\rangle /|-\rangle$ basis. It can continuously run and directly provide the phase offset to a feedback controller, which is used to actively lock the time drift around zero by driving a piezoelectric actuator in $\mathrm{UMZI}_{2}$ (see Methods). Fig. $3 \mathrm{~b}$ shows good system stability with $0.8 \mathrm{~km}$ fiber over one hour. Unlike the well-known automatic polarization compensation (APC) technique ${ }^{31}$, which requires multi-parameter probes, algorithms and feedback controls, and which therefore needs a long adjusting time (several minutes), our phase locking technique only involves one parameter so that it can operate at very high speed, in principle at the speed limit of the phase modulator (nanosecond level). The unique features of simplicity and high speed make our active feedback method a practical solution for long-distance and noisy quantum channels in the real world.

\section{Discussion}

We exploit the time degree of freedom to label polarization quantum states before they are sent and to detect errors when they are received. We do not need to prepare any time-synchronized ancilla photons and have no ancilla-related losses, thus the communication efficiency is enhanced over several orders of magnitude even compared with a single-ancilla-photon scheme. The tolerable error probability is only determined by the signal-to-noise ratio. And our experimental demonstrations of BFE detection was effective for all error probabilities, limited only by the requirement that the signal count rate should exceed the dark count rate. Thus our scheme can deal with error probabilities well beyond the limit of $50 \%$ set by the previous scheme of Chen et $\mathrm{al}^{19}$.

Our experimental method for error detection of BFEs can also be applied to PSE because PSE can be transformed into BFE by a $45^{\circ}$ polarization rotation. In this way, we can detect and filter all PSE instead of BFE. For a specific channel, the functioning of a quantum error detection system can be switched between BFE and PSE depending on which error is dominant. Furthermore, general errors could be detected by using a configuration with four UMZIs. Experimentally, to detect both BFE and PSE, Alice introduces a time delay of $T$ on the $V$ mode of the SP with the first UMZI. After applying a $45^{\circ}$ polarization rotation, she introduces a time delay of $2 T$ on the $V$ mode of the SP with the second UMZI. Bob has the same experimental configuration but the two UMZIs introduce time delays on the $H$ mode of SP. In addition a future realization of such a system robust against general errors could be realized without the need of active phase locking, provided the UMZIs have good phase stability. To this end it should be feasible to use on-chip UMZIs and fast detectors, both of which are within the reach of current techniques ${ }^{32}$.

In summary, we have realized a resource-efficient quantum error detection protocol showing faithful transfer of quantum information over noisy channel. Thanks to the linearity of quantum mechanics, the protocol is state-independent, which makes it suitable for applications in quantum repeaters ${ }^{33}$ and transmitting multi-qubit states, including GHZ states ${ }^{34}$, Cluster states ${ }^{35}$ and $\mathrm{W}$ states $^{36}$ etc. These virtues make quantum error detection promising for the implementation of a long-distance quantum communication network.

\section{Methods}

Coherence recovery between two time bins. For the BFE-free events in central peak, the whole error detection system can be seen as a polarization interferometer. To ensure coherence between $\left|V_{T}\right\rangle$ and $\left|H_{T^{\prime}}\right\rangle$, the time delay between the two arms $\Delta T=(\Delta t+T)-\left(\Delta t+T^{\prime}\right)=T-T^{\prime}$ must be smaller than the coherence time of the SP. The maximum interference visibility can be obtained at $\Delta T=0$. We prepare photons in the $|+\rangle$ state and project onto this state at output. Experimentally, all the photons are collected with SMFs to define the exact spatial mode. A narrowbandwidth filter with FWHM $4 \mathrm{~nm}$ is set in front of each detector to define the exact spectral mode. A prism built on a piezoelectrically controlled linear stage is employed to achieve accurate temporal overlap for the two time bins. A first-order interference of the SP conditioned on herald click from the TP is observed, see Fig. 1d. At the middle of the envelope, by scanning with a step size of $30 \mathrm{~nm}$, we observed phase oscillation with a visibility of $99.2 \%$. The high visibility implies that the spatial and temporal mode of two time bins overlap perfectly on the second PBS of $\mathrm{UMZI}_{2}$.

Active phase locking. We couple the phase probe state $|+\rangle$ (Q-switched, $633 \mathrm{~nm}$ $\mathrm{HeNe}$ laser) in and out of quantum error detection system with dichroic mirrors (DM, T808R633). We adopt achromatic waveplates in the NS to avoid introducing phase difference between HeNe beam and the SP during setting a quantified BFE in the experiment. With same fashion, BFE for HeNe beam is detected and only has influence on the amplitude of output signal. The BFE-induced amplitude fluctuation is further removed by measuring the ratio of $|+\rangle$ to $|-\rangle$. A feedback voltage is amplified and then drives the piezoelectrically controlled linear stage to compensate the drifted phase. In the experiment, we use a computer-based data acquisition system, the feedback adjustment speed is therefore restricted to $\sim 1 \mathrm{~Hz}$. However this limit could be readily overcome by using a commercial PID (proportional-integralderivative) controller.

1. Shor, P. W. Scheme for reducing decoherence in quantum computer memory. Phys. Rev. A 52, R2493-R2496 (1995).

2. Steane, A. M. Error correcting codes in quantum theory. Phys. Rev. Lett. 77, 793 (1996).

3. Bennett, C. H. et al. Mixed-state entanglement and quantum error correction. Phys. Rev. A 54, 3824-3851 (1996).

4. Laflamme, R. et al. Perfect quantum error correcting code. Phys. Rev. Lett. 77, 198-201 (1996).

5. Cory, D. G. et al. Experimental quantum error correction. Phys. Rev. Lett. 81, 2152 (1998).

6. Leung, D. et al. Experimental realization of a two-bit phase damping quantum code. Phys. Rev. A 60, 1924-1943 (1999).

7. Knill, E. et al. Benchmarking quantum computers: the five-qubit error correcting code. Phys. Rev. Lett. 86, 5811 (2001).

8. Chiaverini, J. et al. Realization of quantum error correction. Nature 432, 602 (2004).

9. Pittman, T. B., Jacobs, B. C. \& Franson, J. D. Demonstration of quantum error correction using linear optics. Phys. Rev. A 71, 052332 (2005).

10. Yamamoto, T. et al. Experimental extraction of an entangled photon pair from two identically decohered pairs. Nature 421, 343-346 (2003).

11. Zhao, Z. et al. Experimental realization of entanglement concentration and a quantum repeater. Phys. Rev. Lett. 90, 207901 (2003).

12. Pan, J. W. et al. Experimental entanglement purification of arbitrary unknown states. Nature 423, 417-422 (2003).

13. Zanardi, P. \& Rasetti, M. Noiseless quantum codes. Phys. Rev. Lett. 79, 3306 (1997).

14. Boileau, J. C. et al. Robust polarization-based quantum key distribution over a collective-noise channel. Phys. Rev. Lett. 92, 017901 (2004).

15. Bourennane, M. et al. Decoherence-free quantum information processing with four-photon entangled states. Phys. Rev. Lett. 92, 107901 (2004).

16. Chen, T. Y. et al. Experimental quantum communication without a shared reference frame. Phys. Rev. Lett. 96, 150504 (2006).

17. Yamamoto, T. et al. Robust photonic entanglement distribution by state-independent encoding onto decoherence-free subspace. Nature Photonics 2 , 488-491 (2008).

18. Bouwmeester, D. Bit-flip-error rejection in optical quantum communication. Phys. Rev. A 63, 040301(R) (2001).

19. Chen, Y. A. et al. Experimental quantum error rejection for quantum communication. Phys. Rev. Lett. 96, 220504 (2006).

20. Gisin, N. et al. Error filtration and entanglement purification for quantum communication. Phys. Rev. A 72, 012338 (2005).

21. Lamoureux, L. P. et al. Experimental error filtration for quantum communication over highly noisy channels. Phys. Rev. Lett. 94, 230501 (2005).

22. Gisin, N. et al. Quantum cryptography. Rev. Mod. Phys. 74, 145-151 (2002). 
23. Kalamidas, D. Feasible quantum error detection with linear optics. Physics Letters A 321, 87-93 (2004).

24. Brendel, J., Gisin, N., Tittel, W. \& Zbinden H. Pulsed energy-time entangled twinphoton source for quantum communication. Phys. Rev. Lett. 82, 2594-2597 (1999).

25. Gobby, C., Yuan, Z. L. \& Shields, A. J. Quantum key distribution over $122 \mathrm{~km}$ of standard telecom fiber. Appl. Phys. Lett. 84, 3762 (2004).

26. Franson, J. D. Bell inequality for position and time. Phys. Rev. Lett. 62, 2205-2208 (1989).

27. Nielsen, M. A. \& Chung, I. L. Quantum computation and quantum information, Cambridge University Press (2000).

28. Kwiat, P. G. et al. New high-intensity source of polarization-entangled photon pairs. Phys. Rev. Lett. 75, 4337-4341 (1995).

29. Jin, X. M. et al. Experimental free-space quantum teleportation. Nature Photonics 4, 376-381 (2010).

30. Clauser, J. F., Horne, M., Shimony, A. \& Holt, R. A. Proposed experiment to test local hidden-variable theories. Phys. Rev.Lett. 23, 880 (1969).

31. Peng, C. Z. et al. Experimental Long-Distance Decoy-State Quantum Key Distribution Based on Polarization Encoding. Phys. Rev. Lett. 98, 010505 (2007).

32. Zhang, Q. et al. Distribution of time-energy entanglement over $100 \mathrm{~km}$ fiber using superconducting single photon detectors. Opt. Express 16, 5776 (2008).

33. Briegel, H. J., Dür, W., Cirac, J. I. \& Zoller, P. Quantum repeaters: The role of imperfect local operations in quantum communication. Phys. Rev. Lett. 81, 5932-5935 (1998)

34. Greenberger, D. M. et al. A. Bell's theorem without inequalities. Am. J. Phys. 58, 1131-1143 (1990).

35. Briegel, H. J. \& Raussendorf, R. Persistent entanglement in arrays of interacting particles. Phys. Rev. Lett. 86, 910-913 (2001).
36. Dür, W., Vidal, G. \& Cirac, J. I. Three qubits can be entangled in two inequivalent ways. Phys. Rev. A 62, 062314 (2000).

\section{Acknowledgements}

$\mathrm{XMJ}$ is grateful for insightful discussions with Marco Barbieri, Joshua Nunn, Yu-Ao Chen and Jean-Christian Boileau. This research was supported by the Chinese Academy of Sciences, the National Natural Science Foundation of China (No. 11004183) and China Postdoctoral Science Foundation (No. 201003327). XMJ was financially supported by EU Marie-Curie Fellowship (PIIF-GA-2011-300820).

\section{Author contributions}

X.-M.J. and C.-Z.P. designed and supervised the whole project. X.-M.J., Z.-H.Y., B.Y. and F.Z. performed the experiment. T.Y. and C.-Z.P. designed the electric devices. X.-M.J. analyzed the data and wrote the paper.

\section{Additional information}

Competing financial interests: The authors declare no competing financial interests.

License: This work is licensed under a Creative Commons

Attribution-NonCommercial-ShareAlike 3.0 Unported License. To view a copy of this license, visit http://creativecommons.org/licenses/by-nc-sa/3.0/

How to cite this article: Jin, X.-M. et al. Experimental Quantum Error Detection. Sci. Rep. 2, 626; DOI:10.1038/srep00626 (2012). 